\title{
Aspartic Aminopeptidase Is a Novel Biomarker of Aggressive Chronic Lymphocytic Leukemia
}

\author{
Pramath Kakodkar ${ }^{1} @$, Sanket More ${ }^{1, \dagger}$, Kinga András ${ }^{2, \ddagger}$, Nikos Papakonstantinou ${ }^{3}$, \\ Sharon Kelly ${ }^{1}$, Mohammad Adib Makrooni ${ }^{4}$, Csaba Ortutay ${ }^{2, *}$ and Eva Szegezdi ${ }^{1, *}$ (]) \\ 1 Apoptosis Research Centre, School of Natural Sciences, National University of Ireland Galway, \\ H91 W2TY Galway, Ireland; p.kakodkar1@nuigalway.ie (P.K.); more9.sanket@gmail.com (S.M.); \\ sharon.m.kelly@nuigalway.ie (S.K.) \\ 2 HiDucator Ltd., Erämiehentie 2 E 22, 36200 Kangasala, Finland; andras.kinga89@gmail.com \\ 3 Institute of Applied Biosciences, Centre for Research and Technology Hellas, 57001 Thessaloniki, Greece; \\ n.papakonstantinou83@gmail.com \\ 4 School of Mathematics, Statistics and Applied Mathematics, National University of Ireland Galway, \\ H91 TK33 Galway, Ireland; mohammadadib.makrooni@nuigalway.ie \\ * Correspondence: csaba.ortutay@hiducator.com (C.O.); eva.szegezdi@nuigalway.ie (E.S.) \\ + Current affiliation: Department of Cellular and Molecular Medicine, Laboratory of Cell Death Research and \\ Therapy, University of Leuven (KU Leuven), Herestraat 49, 3000 Leuven, Belgium. \\ $\ddagger$ Current Affiliation: CEU Department of Network and Data Science, Central European University, \\ H1051 Budapest, Hungary.
}

Received: 27 May 2020; Accepted: 8 July 2020; Published: 12 July 2020

\begin{abstract}
Treatment of chronic lymphocytic leukemia has advanced substantially as our understanding of the kinase signal transduction pathways driven by the B cell receptor $(\mathrm{BcR})$ has developed. Particularly, understanding the role of Bruton tyrosine kinase and phosphatidyl inositol 3 kinase delta in driving prosurvival signal transduction in chronic lymphocytic leukemia (CLL) cells and their targeting with pharmacological inhibitors (ibrutinib and idelalisib, respectively) has improved patient outcomes significantly. The kinase signaling pathway induced by the $\mathrm{BcR}$ is highly complex and has multiple interconnecting branches mediated by tyrosine and serine/threonine kinases activated downstream of the BcR. There is a high level of redundancy in the biological responses, with several $\mathrm{BcR}$-signaling kinases driving nuclear factor kappa $\mathrm{B}$ activation or inducing antiapoptotic Bcl-2 genes. Accordingly, common gene targets of BcR-signaling kinases may serve as biomarkers indicating enhanced BCR-signaling and aggressive disease progression. This study used a gene expression correlation analysis of malignant B cell lines and primary CLL cells to identify genes whose expression correlated with BCR-signaling kinases overexpressed and/or overactivated in CLL, namely: AKT1, AKT2, BTK, MAPK1, MAPK3, PI3KCD and ZAP70. The analysis identified a 32-gene signature with a strong prognostic potential and DNPEP, the gene coding for aspartic aminopeptidase, as a predictor of aggressive CLL. DNPEP gene expression correlated with MAPK3, PI3KCD, and ZAP70 expression and, in the primary CLL test dataset, showed a strong prognostic potential. The inhibition of DNPEP with a pharmacological inhibitor enhanced the cytotoxic potential of idelalisib and ibrutinib, indicating a biological functionality of DNPEP in CLL. DNPEP, as an aminopeptidase, contributes to the maintenance of the free amino acid pool in CLL cells found to be an essential process for the survival of many cancer cell types, and thus, these results warrant further research into the exploitation of aminopeptidase inhibitors in the treatment of drug-resistant CLL.
\end{abstract}

Keywords: chronic lymphocytic leukemia (CLL); DNPEP; biomarker; B-cell receptor (BcR) signaling; Bruton tyrosine kinase; phosphatidyl inositol-3 kinase; Akt; aspartic aminopeptidase; mitogen-activated protein kinase (MAPK) 


\section{Introduction}

Chronic lymphocytic leukemia (CLL) is a malignancy of mature B cells. With more than 11,000 cases diagnosed every year in Europe and 15,000 in the USA, CLL is the most common type of leukemia diagnosed in the Western world [1]. CLL displays remarkable clinical heterogeneity, with many patients experiencing an indolent disease state and never requiring treatment, contrasting others who develop an aggressive disease needing treatment soon after diagnosis. Therefore, the accurate identification of patients with the aggressive disease phenotype is important.

CLL is characterized by the clonal expansion of $\mathrm{CD}^{+} \mathrm{CD} 23^{+} \mathrm{B}$ cells [2], which is driven by the accumulation of cell-intrinsic aberrations, as well as by the enhancement of $\mathrm{B}$ cell receptor $(\mathrm{BcR})$ signaling. Approximately $55-60 \%$ of CLL patients display somatic hypermutation (SHM) in the rearranged clonotypic immunoglobulin heavy variable (IgHV) genes of the $\mathrm{BcR}$ (mutated CLL, M-CLL), while the remainder carry IgHV genes with minimal or no SHM (unmutated CLL, U-CLL). M-CLL is generally associated with a favorable prognosis, contrasting U-CLL, which typically exhibits an aggressive clinical course and adverse prognosis [3].

Engagement of the $\mathrm{BcR}$ with antigens triggers a cascade of kinase activation driving the activation of $B$ cells. Upon activation of the BcR, the first kinases activated are the spleen tyrosine kinase (Syk) and the SRC kinase Lyn, which are recruited to the BcR complex and phosphorylate its immunoreceptor tyrosine-based activation motifs (ITAMs), namely Ig $\alpha$ (CD79a) and Ig $\beta$ (CD79b) [4]. Phosphorylation of $\operatorname{Ig} \alpha$ and $\operatorname{Ig} \beta$ at the ITAMs allows for the recruitment of adaptor proteins and further kinases, such as Bruton's tyrosine kinase (BTK) and phosphatidylinositol 3-kinase (PI3K), driving the downstream activation of the prosurvival AKT pathway, followed by mTOR (mammalian target of rapamycin), NF-KB (nuclear factor kappa-light-chain-enhancer of activated B cells), and/or ERK (extracellular signal-regulated kinase) activation [4]. Studies on CLL cells demonstrated that BCR signaling is enhanced by a Zeta-associated protein of $70 \mathrm{kDa}$ (ZAP70). ZAP70 is a homolog of Syk, first identified as the apical kinase in the $\mathrm{T}$ cell receptor-mediated signal transduction pathway. The constitutive activity of ZAP70 contributes to the untethered activation of downstream signaling kinases, leading to enhanced BCR signaling [5]. High levels of ZAP70 protein expression correlate with higher disease stages, i.e., Binet stage B or C [6]. Expression of the ZAP70 protein is also an established surrogate marker for the IgHV gene SHM status, whereby U-CLL correlates with a 5.5-fold more ZAP70 protein concentration $[6,7]$.

Until recently, the mainstream management of CLL consisted of a combinatorial drug regimen of genotoxic agents, such as the alkylating agent cyclophosphamide and purine analogs (fludarabine) in combination with the anti-CD20 monoclonal antibody, Rituximab. However, over time, many CLL patients developed resistance against the concurrent genotoxic and cytotoxic stresses triggered by these agents. This evolution in resistance can be abstracted to the production of trophic factors released by the lymph node microenvironment and activation of BcR-signaling kinases, resulting in compensatory prosurvival signal transduction, such as induction of the antiapoptotic BCL2 [8,9] protein family.

Of the BcR-signaling kinases, the most commonly used biomarker for aggressive CLL is ZAP70. However, not all patients with a high ZAP70 expression have an aggressive disease, and, vice versa, not all patients with a low level of ZAP70 have an indolent disease. For example, in approximately $25 \%$ of patients, discordance has been observed between the ZAP70 expression and IgHV gene SHM status [10]. This observed discordance can be explained by the presence of additional genetic high-risk features, such as chromosomal deletions (e.g., 11q or 17p) [10]. Furthermore, while both 17p deletion and ZAP70 expression predict the aggressive disease, the 17p deletion falls within the ZAP70-negative patient group [10]. Moreover, there is also a high variation in ZAP70 expression depending on where the sample was collected from within the patient, i.e., peripheral blood, bone marrow, and lymph node specimen [11].

ZAP70 enhances BcR signaling by facilitating the recruitment of other kinases to the BcR complex, such as Syk, rather than via its own kinase activity [12]. Moreover, the expression of ZAP70 enhances and prolongs the activation of several key mediators of BcR signaling, such as Syk, ERK, and Akt, 
and decreases the rate of ligand-mediated BcR internalization. ZAP70 is also found to interact with other signaling kinases and adaptor proteins like PI3K and Shc, respectively [12]. Thus, the constitutive activation of kinases like Syk, PI3K, Akt, Btk, and ERK in response to the ZAP70 expression further drives $\mathrm{BCR}$ signaling, enhancing prosurvival signaling and the consequent resistance against cellular stresses triggered by chemotherapeutics. Drugs targeting key BcR-signaling kinases, particularly Btk and PI3K, have shown great success in treating patients with the aggressive disease. Especially, two oral kinase inhibitors, idelalisib (PI3K inhibitor) and ibrutinib (BTK inhibitor), have changed the standard of care for CLL patients in both the relapsed and frontline settings [13]. Despite the outstanding efficacy observed with these agents, patients still relapse, and some patients fail to respond, which is likely, since many patients have an increased activity of multiple BcR-signaling kinases, and, due to their complex interrelationship, the inhibition of only one of these kinases triggers a compensatory mechanism via other kinases and a consequent development of resistance.

In this study, we aimed to identify common or shared targets of BcR-signaling kinases as better biomarkers of aggressive CLL and potential targets to reverse drug resistance using a gene co-expression network analysis. We hypothesize that signal transduction pathways driven by the lymphoid microenvironment-activated kinases converge on common effectors. The identification of these effectors can provide novel and early biomarkers, aggressive CLL, and possible drug targets to sensitize drug-resistant CLL cells.

\section{Results}

\subsection{Identification BCR Signaling Kinase-Correlating Genes}

While ZAP70 contributes to enhanced BcR-signaling via multiple mechanisms, now it is clear that additional pathways, activated by either oncogenic transformation triggering cellular stress pathways or the lymphoid microenvironment, play an important role. Thus, in order to identify biomarkers of enhanced BcR-signaling, we identified genes whose expressions correlated with multiple BcR-signaling kinases, namely: ZAP70, AKT1, AKT2, BTK, MAPK1, MAPK3, and PI3KCD, using bi-weight mid-correlation analysis. For the analysis, $14 \mathrm{~B}$ cell gene expression microarray datasets were obtained from ArrayExpress at the EBI (European Bioinformatics Institute) and Gene Expression Omnibus (GEO) at NCBI. A description of the studies and the number of genes and samples in the datasets are summarized in Table S1. The bi-weight mid-correlation values were first individually calculated for the 14 datasets. Then, a threshold value of 0.5 was set to select the highly correlating genes. Of these genes, there were 1262 whose expressions correlated with ZAP70 and at least one other BcR-signaling kinase in at least five datasets (Figure 1A,B). From this list, the genes that showed correlations with multiple kinases were selected out for further analysis. The final selection contained 32 genes whose expressions correlated with ZAP70 and a minimum of two other BcR-signaling kinases (Table S2). Of these 32 genes, the ones that correlated with ZAP70 and AKT1/2 expressions also correlated with MAPK3 and PI3KCD expressions but not with BTK (Figure 1C,D). Interestingly, there was a relatively small overlap between $A K T 1 / 2$ and PI3KCD co-expressed genes. Many of the genes that correlated with ZAP70 and PI3KCD also showed co-expressions with MAPK3 but not with $A K T 1 / 2$ or $B T K$, indicating that the gene expressional programs associated with different $B c R$-signaling kinases are diverse and only partially overlapping (Figure 1C,D).

A network analysis found that 28 of the 32 genes formed a closely connected, minimal network, clustering around four main nodes: HNF4A (hepatocyte nuclear factor 4 alpha), EED (embryonic ectoderm development), ELAVL1 (ELAV-like RNA binding protein 1), and MAPK1/3 and that the 32-gene signature reports on the activity of these four genes/pathways. This well-interlinked signaling network (Figure 1E) contains nodes already known to have a role in CLL, such as EZH2 and NF-kB, and also identified new pathways not well-associated with CLL (HNF4A and ELAVL1 nodes) [14-16]. 

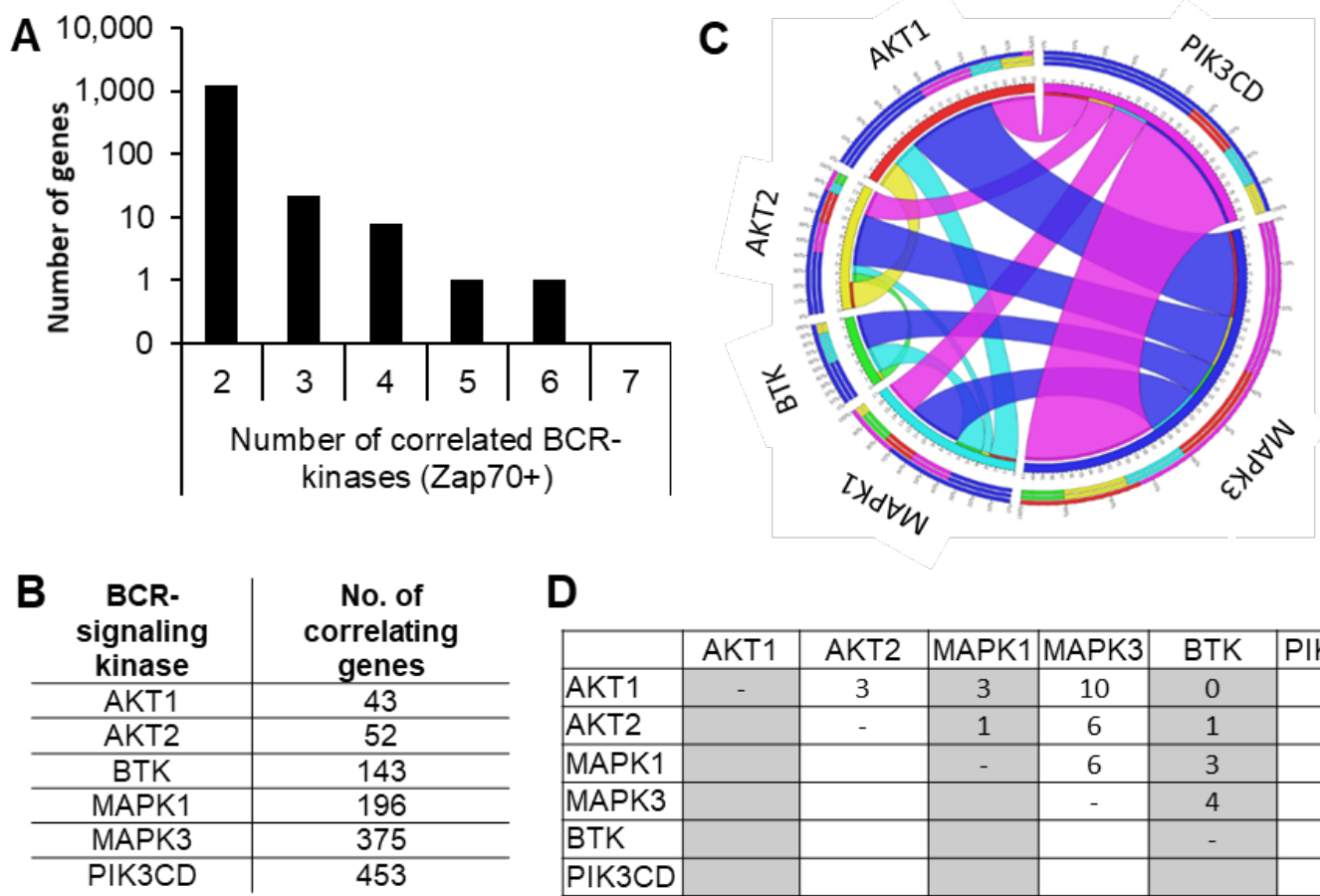

D
\begin{tabular}{|l|c|c|c|c|c|c|}
\hline & AKT1 & AKT2 & MAPK1 & MAPK3 & BTK & PIK3CD \\
\hline AKT1 & - & 3 & 3 & 10 & 0 & 5 \\
\hline AKT2 & & - & 1 & 6 & 1 & 3 \\
\hline MAPK1 & & & - & 6 & 3 & 4 \\
\hline MAPK3 & & & & - & 4 & 15 \\
\hline BTK & & & & & - & 0 \\
\hline PIK3CD & & & & & & - \\
\hline
\end{tabular}

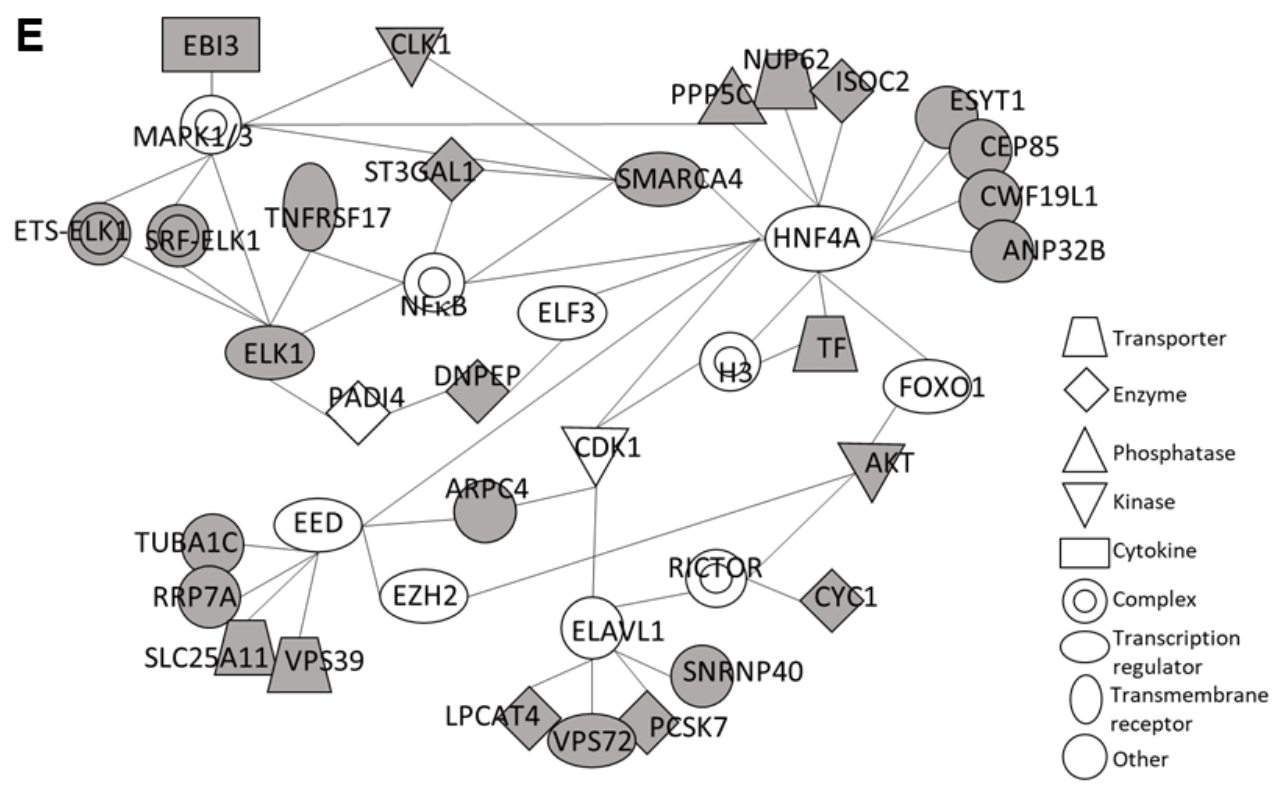

Figure 1. Partial integration and diversity in gene expressional programs that drive the expressions of BCR-signaling kinases. (A) Total number of genes whose expressions correlated with that of ZAP70 and at least one other BCR-signaling kinase in at least five datasets. (B) The number of correlating genes identified for each BCR-signaling kinase. (C) Circos plot showing the distribution of common targets of BCR-signaling kinase pairs. (D) Matrix representation of the number of genes that are common correlating genes of BCR-signaling kinase pairs. (E) Interaction network of the 32 genes identified. Ingenuity pathway analysis was carried out to identify gene networks the 32 BCR-signaling kinase co-expressed genes reported on. Grey-shaded genes are the identified BCR-kinase correlating genes.

\subsection{DNPEP Is a Prognostic Marker of Aggressive CLL}

Further analysis was directed towards validating the prognostic power of the identified genes by analyzing the time to treatment and overall survival responses using an independent transcriptomic 
dataset of 107 CLL patients [17]. For both analyses, the hazard ratio associated with ZAP70 mRNA expression was used as a baseline for comparison. Regarding time-to-treatment, a high ZAP70 mRNA expression was associated with a hazard ratio of 1.45 (of note, the clinically used Zap70 expression measure, the percentage of Zap70 protein-expressing cells, was not recorded in the dataset; thus, we used the mRNA expression values). As a measure of their prognostic potential, the HR values associated with the time to treatment for the 32 genes were determined individually (Figure 2A), as well as together (Figure 2B). When analyzed together, the 32-gene set could clearly segregate low and high-risk groups with an HR value of 24.49 (Figure 2B). When the 32 genes were analyzed individually, 8 out of the 32 genes (DNPEP, ARPC4, SMARCA4, TUBA1C, PSMD13, VPS39, EBI3, and ESYT1) were associated with an HR higher that of ZAP70 (Figure 2A), and combined, these eight genes could segregate the patients into high and low-risk groups with an HR of 2.99 (Figure 2C). Additionally, the contribution of each gene to the combined HR was assessed by the iterative removal of each gene, one at a time, from the 32-gene pool (Figure S1A). The strongest eight contributors to the HR were PCSK7, CYC1, VPS72, PSMD13, RPL38, SLC25A11, DNPEP, and ISOC2. This eight-gene signature was associated with an HR value for a time to treatment of 3.47 (Figure S1C).
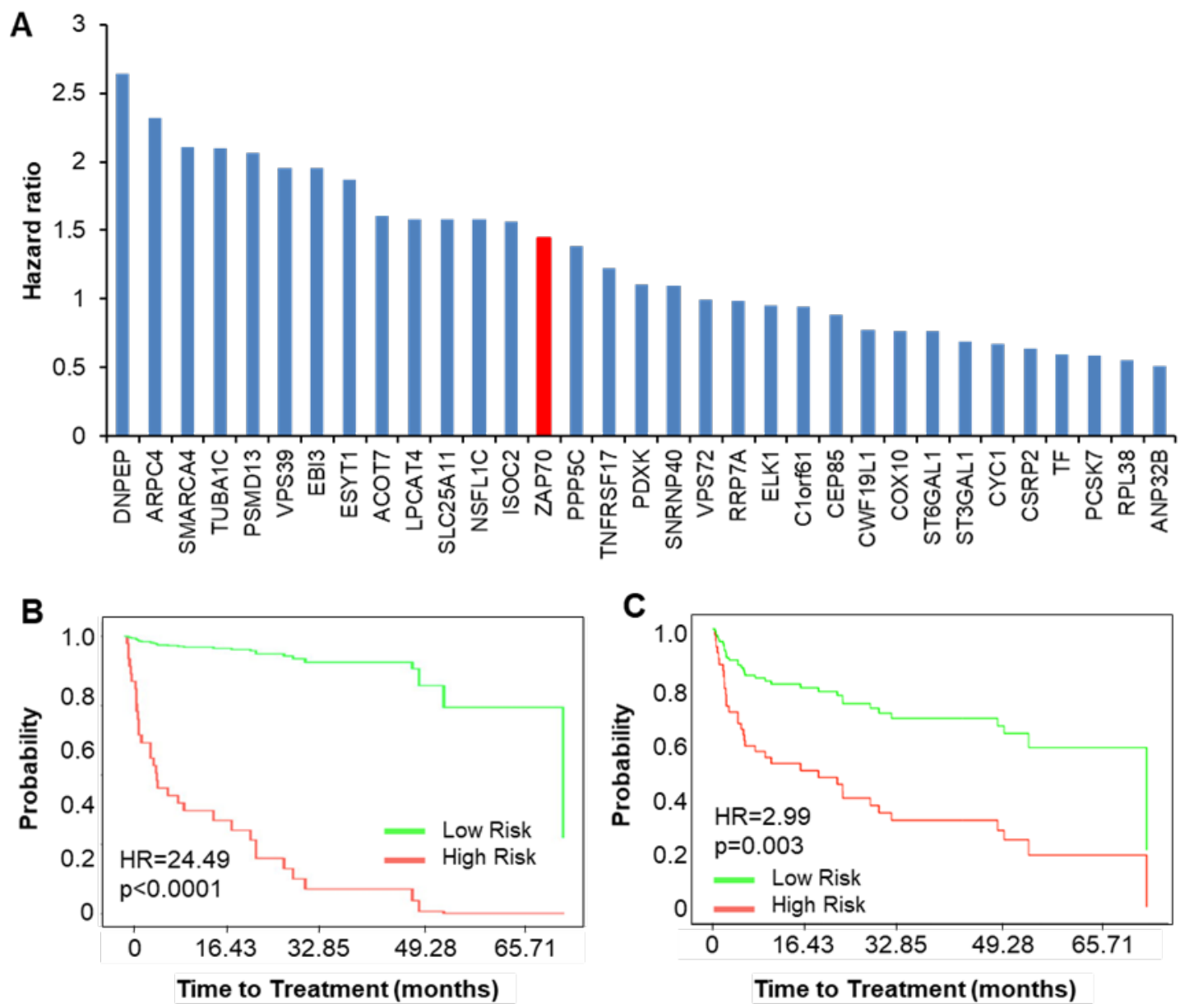

Figure 2. Prognostic potential of genes correlating with BCR-signaling kinases regarding the time to treatment. (A) Bar graph showing hazard ratios associated with each of the 32 genes in the panel. The hazard ratio (HR) of ZAP70 (red bar) is added as a reference. (B,C) Kaplan Meier plots showing time-to-treatment probability curves for the 32 genes together $(\mathbf{B})$ and the combination of the eight genes with the highest HR values (from graph in part A, (CI: 1.44 to 6.21)). The $y$-axis represents the estimate probabilities, and the $x$-axis represents the time to treatment (months).

The same analysis as above was carried out for the overall survival. The reference ZAP70 expression was associated with an HR of 1.94, while the hazard ratio associated with the combination of the $32 \mathrm{BCR}$ 
kinase-correlating genes was 32.93 (Figure 3B). An analysis of the 32 genes individually (Figure 3A) revealed eight genes associated with a high HR, and the combination of these eight genes could segregate the patients into high and low-risk groups, with an HR of 6.87 (Figure 3C). This cluster of eight genes consisted of PSMD13, DNPEP, TUBA1C, RPL38, ISOC2, ESYT1, SMARCA4, and ST6GAL1. The contribution of each gene to the combined HR was also assessed by the iterative removal of each gene, one at a time, from the 32-gene pool (Figure S1B). The strongest eight contributors of this analysis included ARPC4, EBI3, RPP7A, PCSK7, DNPEP, Corf61, PDXK, and CWF19L1, and, combined, these eight genes could segregate the CLL patients into high and low-risk groups with an HR of 7.47 (Figure S1D).
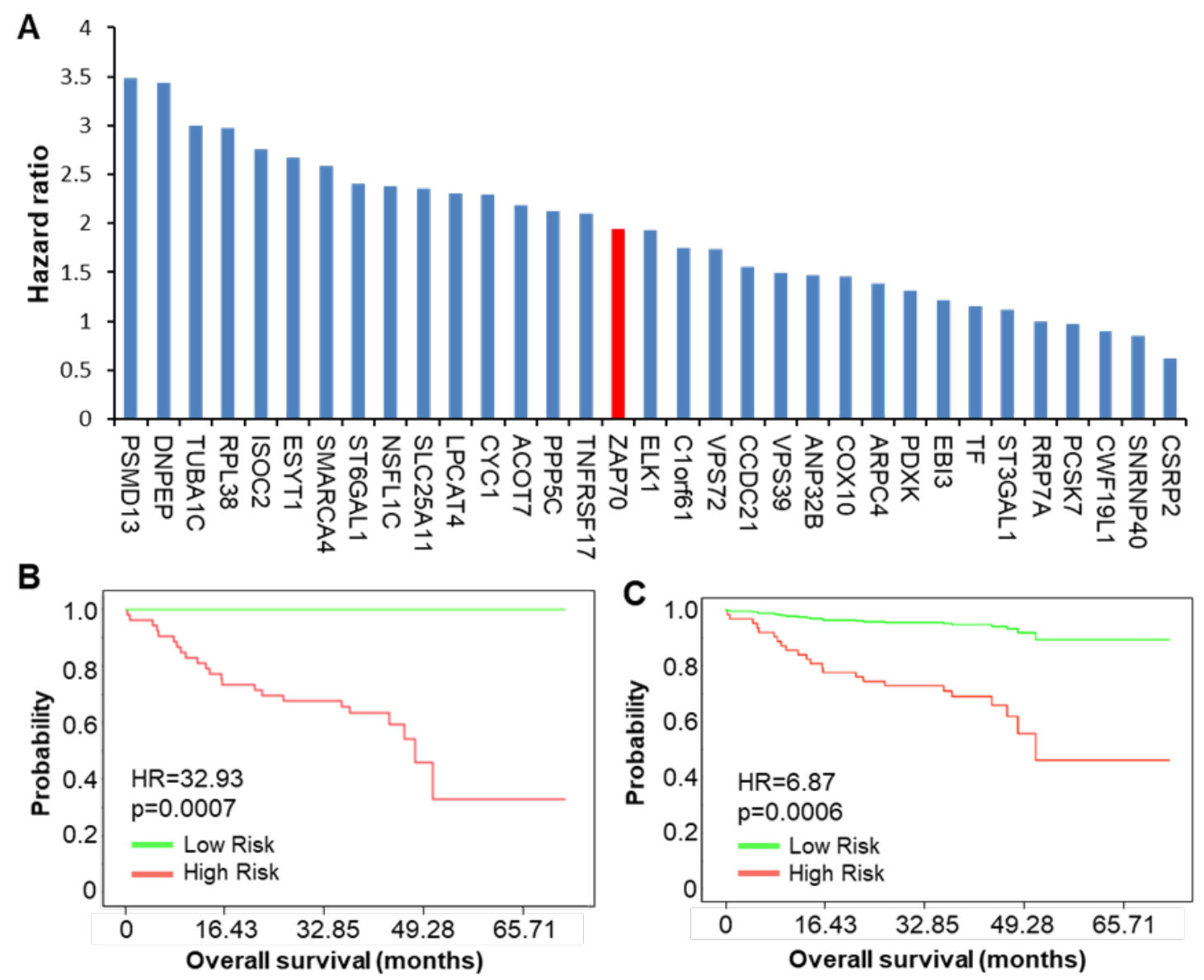

Figure 3. Prognostic potential of genes correlating with BCR-signaling kinases regarding the overall survival. (A) Bar graph showing the hazard ratios for the overall survival associated with each of the 32 genes in the panel. The HR of ZAP70 (red bar) is added as a reference. (B,C) Kaplan Meier plots showing the overall survival probability curves for the 32 genes together (CI: 4.4 to 246.3) (B) and the combination of the eight genes with the highest HR value (from graph in part A, (CI: 2.28 to 20.67, $p=0.0006)$ ). The $y$-axis represents the estimate probabilities, and the $x$-axis represents the time to treatment (months).

Overall, from the analyses at the individual gene level, four genes were found to correlate with both the time-to-treatment and overall survival: DNPEP, PSMD13, ESYT1, and SMARCA4. From the reciprocal analysis (iteratively removing one gene out of the pool of 32), only DNPEP was common between the time-to-treatment and overall survival, and DNPEP emerged as the common gene across the four analyses. 


\subsection{DNPEP as a Prognostic Marker for CLL}

By dichotomizing the patients from the Herold dataset into high- and low-DNPEP expressing groups based on the median DNPEP expression, patients with high DNPEP expressions tended to have a shorter time to treatment, as well as overall survival probability (Figure $4 \mathrm{~A}, \mathrm{~B}$ ). To further validate $D N P E P$ as a marker for prognosis, DNPEP transcript levels were measured in 34 primary CLL patient samples with real-time quantitative RT-PCR, and a correlation with Rai and Binet staging was determined. The clinical information of the samples is summarized in Table S3. The DNPEP mRNA expression correlated with Rai staging, with the DNPEP expression of the asymptomatic stage (0) being significantly lower than a high risk (III-IV) $(p=0.0019)$. Similarly, DNPEP transcript levels of medium-risk (I-II) and high-risk (III-IV) groups were also significantly different ( $p=0.0014$, Figure $4 \mathrm{C}$ ). The DNPEP expression also correlated with Binet stages, with a high DNPEP expression detected in the intermediate and high-risk groups (B and C stages, $p=0.024$, Figure $4 \mathrm{D}$ ).
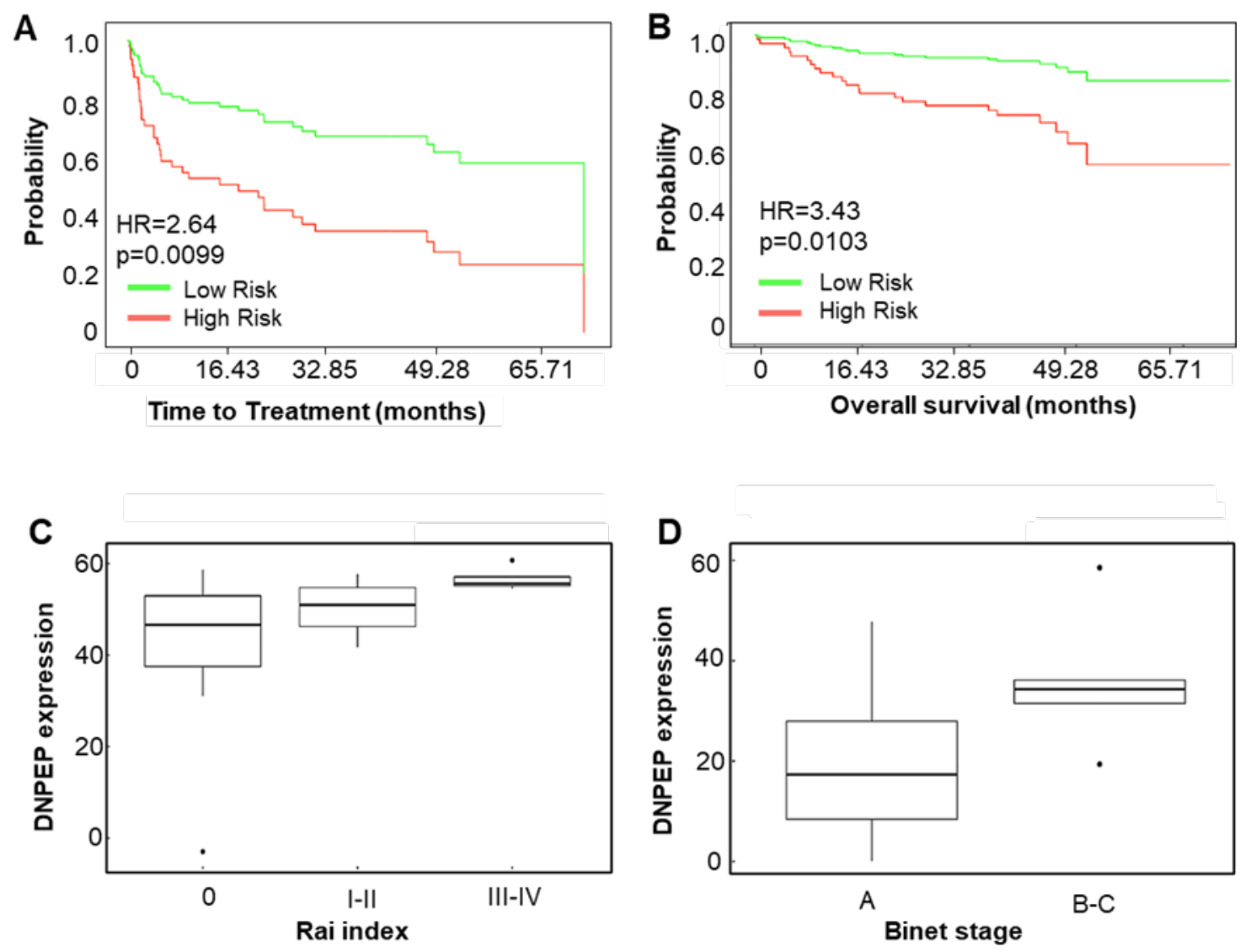

Figure 4. Prognostic potential of DNPEP. Kaplan-Meier plots for time-to-treatment (A) and overall survival (B) for DNPEP expression (CI: 1.26 to 5.54 and CI: 1.34 to 8.79 for A and B, respectively). (C,D) Correlation between DNPEP expression and disease stage. Patient samples were divided into low risk and high risk based on the median DNPEP mRNA expression, and the time to treatment and overall survival probabilities were graphed. The DNPEP mRNA expression was determined by quantitative real-time (RT)-PCR from 34 chronic lymphocytic leukemia (CLL) patient RNA samples. The box plots show the DNPEP expressions by Rai (C) and Binet (D) staging. (C) The difference between the DNPEP transcript levels of the Rai medium-risk (I-II) and high-risk (III-IV) groups and between the asymptomatic stage (Rai 0) and high risk (III-IV), as well as (D) between the early stage (Binet A) and late metastatic stages (Binet B and C), were significant $(p=0.0019, p=0.0014$, and $p=0.024$, respectively).

To determine whether DNPEP is an independent prognostic marker, it was compared to the immunoglobulin variable heavy chain $(\mathrm{IGVH})$ gene mutational status and $17 \mathrm{p} 13$ deletion in a 
multivariate (Cox) analysis looking at the time to treatment and overall survival using the Herold dataset [17]. In the database, $48 \%$ of the patients had mutated IGVH, $45 \%$ had unmutated IGVH, and $7 \%$ was unknown, while $8 \%$ of the patients carried 17 p13 deletion. A high DNPEP expression (based on the median expression) was a significant prognostic factor in the univariate analysis, both for the time to treatment and overall survival ( $p=0.009$ and $p=0.01$, respectively). In the multivariate Cox regression analysis, a high DNPEP expression remained prognostic for the time to treatment after adjusting for the IGVH mutational status and 17p13 deletion but not for the overall survival ( $p=0.032$ and $p=0.224$, respectively).

\subsection{Inhibition of DNPEP Increases Sensitivity to PI3K- and BTK-Inhibitions}

In order to investigate whether DNPEP is only a biomarker or a possible therapeutic target, a recently developed inhibitor against DNPEP was used [18,19]. The DNPEP gene codes for an N-terminal peptidase selective for aspartic acids. The inhibitor, DI93293, used in our study could target two aminopeptidases, DNPEP and the closely related ENPEP (glutamine aminopeptidase). The inhibitor inhibited the peptidase activity of recombinant DNPEP by $95.0 \% \pm 1.4 \%$ [18] and by $88 \%$ in Mec-1 CLL cell lysate (at $12.5 \mu \mathrm{M}$ and $25 \mu \mathrm{M}$, respectively, Figure S2). The $\mathrm{IC}_{50}$ value of the inhibitor was $1.4 \mu \mathrm{M}[18]$.

To test the effect of the DNPEP inhibition on CLL cell survival and drug resistance, Mec-1 CLL cells were cultured alone or on a mesenchymal stromal cell layer expressing the CD40 ligand (CD40L) as a model of the CLL microenvironment. After $24 \mathrm{~h}$ of culture, the cells were exposed to a dosage of DI93293 alone or with the Bcl-2/Bcl- $\mathrm{X}_{\mathrm{L}}$ inhibitor, ABT737, for $48 \mathrm{~h}$, and the induction of cell deaths in Mec-1 cells were determined with annexin $V$ staining (after the exclusion of stromal cells based on their green fluorescent labels). The inhibition of DNPEP alone did not induce any cell deaths but had a modest potentiating effect on ABT737-induced cytotoxicity shown by the CI indices of lower than 1 for all drug dose combinations (calculated by the Chou-Talalay method; Figure 5A, tables under the plots). Stromal support of the CLL cells mostly affected the ABT737 sensitivity, but the potentiating effect of DI93293 was retained (Figure 5B).

The effect of DNPEP inhibition on the drug sensitivity of primary, patient-derived CLL cells was also tested using ABT737 and two kinase inhibitors, the PI3K inhibitor, idelalisib, and the BTK inhibitor, ibrutinib (Figure 5C-E). Mononuclear cells isolated from five patients were cultured with bone marrow mesenchymal stromal cell support for $24 \mathrm{~h}$ before exposing the cultures to DI93293 alone or in combination with the above drugs for $48 \mathrm{~h}$. The induction of cell death was determined with To-Pro3 staining in the CLL cell population. We found that the inhibition of DNPEP potentiated cell deaths induced by both idelalisib and ibrutinib but not ABT737 (Figure 5D,E). 
A

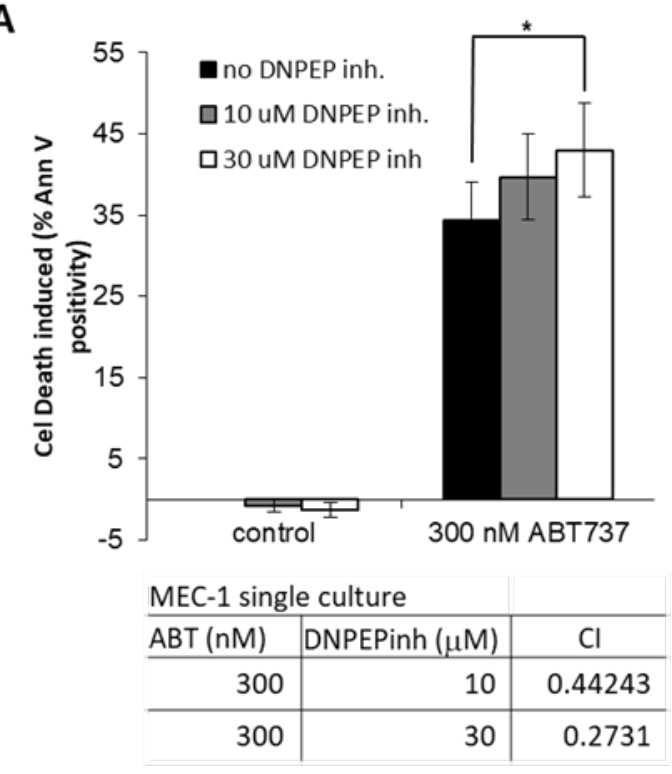

B

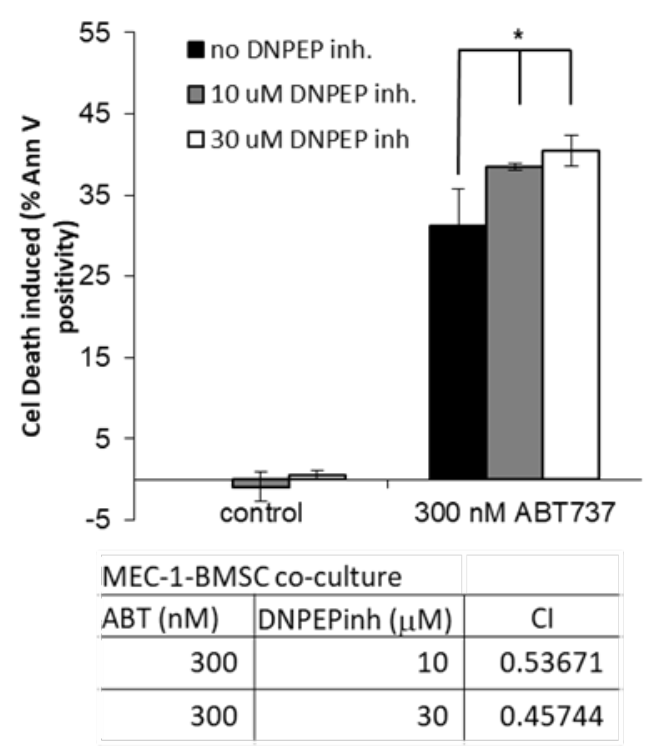

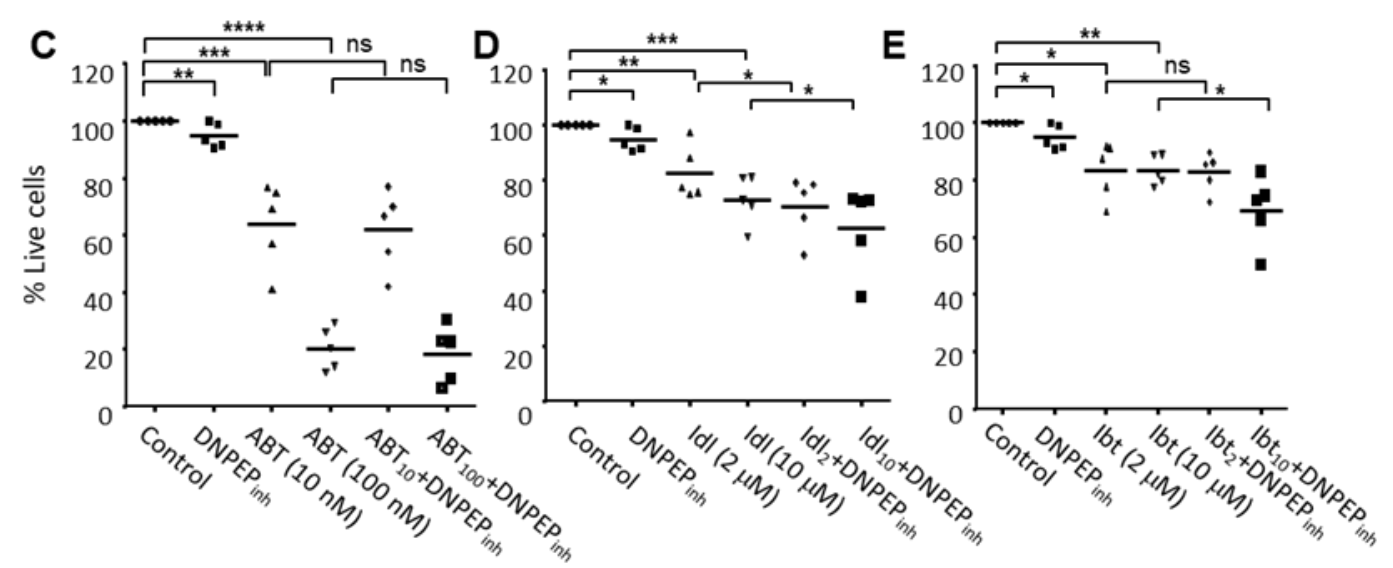

Figure 5. (A) The inhibition of DNPEP activity potentiates AB737-induced CLL cell death. Mec-1 CLL cells were treated with $300 \mathrm{nM}$ of the BH3-mimetic (ABT737) and with the indicated doses of the developmental DNPEP inhibitor DI93293 for $48 \mathrm{~h}$-after which, the percentage of dead cells was determined with the annexin $\mathrm{V}$ assay. ${ }^{*} p<0.05$. (B) The potentiating effect of DNPEP inhibition is retained in a microenvironment-modeling culture of CLL. Mec-1 cells were seeded on a monolayer of CD40L-expressing bone marrow stromal cells for $24 \mathrm{~h}$ before exposing them to 300-nM ABT737 and indicated doses of the DNPEP inhibitor DI 93293 for $48 \mathrm{~h}$. Cell deaths induced were determined with the annexin $\mathrm{V}$ assay. The tables under the graphs of parts $(\mathbf{A}, \mathbf{B})$ list the combination indices calculated using the Chou-Talalay method (Compusyn). (C-E) The potentiating effect with DNPEP inhibition on primary CLL cells was tested on ABT737 (ABT), the PI3K inhibitor (idelalisib. Idl), and the BTK inhibitor (ibrutinib, Ibt). Mononuclear cells isolated from 5 patients were cultured with bone marrow mesenchymal stromal cell support for $24 \mathrm{~h}$ before exposing the cultures to DI93293 alone or in combination with the above drugs for $48 \mathrm{~h}$. The induction of cell death was measured with To-Pro3 staining in the CLL cell population. $p$-values: ${ }^{*} p<0.05,{ }^{* *} p<0.01,{ }^{* * *} p<0.001$, and ${ }^{* * * *} p<0.0001$.

\section{Discussion}

The identification of biomarkers able to indicate the activity of several BcR-linked kinases would improve patient stratification. By using a bi-weight gene expression correlation analysis, we identified genes whose expressions correlated with several BCR-signaling kinases. Genes whose expression could predict patient prognosis correlated with MAPK1 and/or MAPK3, PI3KCD, and ZAP70 and 
PI3KCD, indicating that the gene expressional program's driving expressions of BcR-signaling kinases are diverse and only partially overlapping.

By selecting the genes whose expressions correlated with ZAP70 plus two other BCR-signaling kinase gene, we identified a 32-gene panel. The 32-gene panel could prognose the time to treatment and overall survival probability with very high HR values of 24.49 and 32.93 , respectively. The reduction of the number of genes by selecting the top quartile from each analysis, while still producing a prognostic gene set, was nearly not as robust as the 32 genes together, highlighting again that the BCR-induced kinase signaling pathways are only partially overlapping. One gene, aspartic aminopeptidase (DNPEP), showed a high HR value and consistently appeared in all analyses. This biomarker can predict and stratify the risk when CLL patients develop the progressive disease, requiring therapy. An analysis of the DNPEP expression in combination with the $\mathrm{IGV}_{\mathrm{H}}$ mutation status could be used as a prognostic tool to detect when CLL patients develop the progressive disease and, therefore, when treatment would be required.

$D N P E P$, a member of the M18 peptidase family, is a zinc/manganese-containing metallopeptidase expressed in the cytosol [13]. DNPEP cleaves N-terminal aspartate residues from proteins and peptides. Aminopeptidases execute the final step of intracellular protein degradation by trimming or fully degrading peptides produced by the ubiquitin-proteasome pathway. Partial peptide degradation by aminopeptidases may be used to generate MHC-presented peptide antigens, or, after full hydrolysis, the generated free amino acids can be reutilized by the CLL cells for protein synthesis.

Although, to date, little is known about the physiological functions of DNPEP, the enhanced expressions of other aminopeptidases in cancer are long known. For example, pancreatic cancer, lymphoma, and leukemia patients have increased leucine aminopeptidase (LAP) activity. Aminopeptidase N (APN) is another type of aminopeptidase implicated in human cancers, such as thyroid cancer [20], ovarian carcinoma [21], breast [22,23], and colon cancer [24]. Moreover, APN expression was shown to correlate with poor survival, as well as decreased disease-free survival in colon cancer [24].

Further indicating the potential role of aminopeptidases in cancer, the aminopeptidase inhibitor [25] bestatin showed efficacy in lung cancer [26]. New aminopeptidase inhibitors are also emerging, with the best-known example of the prodrug, tosedostat, which is currently in phase II clinical trials for acute myeloid leukemia [25,27].

These results show that aminopeptidases are not only biomarkers of various cancers, but they have a biological functionality in cancer. The free amino acids produced by aminopeptidases support protein synthesis, which some cancers strongly rely on. Accordingly, the inhibition of aminopeptidases by CHR79888 (the active metabolite of tosedostat) induced a typical amino acid deprivation response (AADR) in HL-60 AML cells, including the inhibition of mTOR phosphorylation, reducing the protein synthesis and accumulation of intracellular peptides [27]. New emerging results, which show that leukemic cells are sensitive to amino acid deprivation, further support the rationale of aminopeptidase inhibitors as cancer therapeutics [28].

\section{Materials and Methods}

\subsection{B Cell Transcriptomic Datasets and Preprocessing}

B cell transcriptomic datasets were obtained from two main public databases, the ArrayExpress at the EBI (European Bioinformatics Institute, https://www.ebi.ac.uk/arrayexpress/) and the Gene Expression Omnibus (GEO) at NCBI (National Centre for Biotechnology Information, https: //www.ncbi.nlm.nih.gov/geo/). Fourteen B cell transcriptomic datasets were selected. Preprocessing of gene expression datasets included normalization and annotation of the datasets using the open-source programming language " $R$ ". Datasets from single-channel microarray datasets were normalized using the Robust Multi-Array Average (RMA) method, while two-channel microarray datasets were 
normalized using the locally weighted scatterplot smoothing (LOESS) method. These normalized datasets were annotated by using BiomaRt and Bioconductor packages.

\subsection{Weighted Gene Co-Expression Network Analysis}

For calculating correlation values between BCR-signaling kinase genes and genes in microarray datasets, the WGCNA package [29] was used, which implements the necessary functions for correlation calculation, gene selection, cluster identification, and network construction. Bi-weight mid-correlation was used to identify genes whose expression correlates with BCR-signaling kinases. The gene list obtained was filtered by applying an absolute threshold and ranking the genes in descending order by the number of datasets the gene was present in for each kinase.

\subsection{Statistical Analyses}

For time-to-treatment and overall survival analyses, an independent transcriptomic dataset from 107 CLL samples analyzed on Affymetrix HG-U133 Plus 2.0 chips was used [17,30]. For analyses where the combined effect of several genes was assessed, the sample set was divided into two equal-sized risk groups by ranking and splitting the samples at the median according to their estimated prognostic index (risk score). The prognostic index was calculated based on the beta coefficients multiplied by gene expression values using Cox proportional hazard regression: $\left(\mathrm{h}_{i}(\mathrm{t}) / \mathrm{h}_{0}(\mathrm{t})=\exp \left(\beta_{1} x_{1}+\beta_{2} x_{2}+\ldots \beta_{i} x_{i}\right)\right.$, $\mathrm{h}_{i}$ is the hazard of the $\mathrm{i}$-th individual, $\mathrm{h}_{0}$ is the baseline hazard function, exp is the exponent function, $x_{i}$ is the expression value and the $\beta_{i}$ can obtained from the Cox fitting and the term within the exp function is the prognostic index or risk score. In case a single gene was analyzed, the dataset was divided into two groups based on the median expression level of the gene of interest. Cox proportional hazards models to model the probability of survival and the time to treatment were used where the DNPEP expression (as a binary variable indicating low and high-risk individuals based on the median DNPEP expression) was the only predictor. For multivariate analysis, Cox proportional hazards models were used to model the probability of the survival and treatment with three predictors: IGV $_{\mathrm{H}}$ mutational status, p17 mutational status, and DNPEP expression. The Coxph function in the Survival package in $\mathrm{R}$ was used to compute the Cox Proportional Hazard regression models and obtain the hazard ratios. The dependent variable, which is a survival object in this linear model, was created using the Surv function in Survival package. Additionally, the Survminer package in $\mathrm{R}$ was used to plot the survival curves. The dynamic nomogram of these models, along with the underlying model summaries, have been provided by the DynNom package [31] in R: https:/adibmakrooni.shinyapps.io/cxmod10/, https://adibmakrooni.shinyapps.io/cxmod4/, https:/adibmakrooni.shinyapps.io/cxmod1/, and https: //adibmakrooni.shinyapps.io/cxmod2/. The expression values of DNPEP in the primary CLL samples were analyzed in the patients grouped by the Rai and Binet clinical CLL stages. Regarding the Rai stages, stage 0 patients $(n=20)$ were handled as low risk, stage I-II patients $(n=10)$ as intermediate, and stage III-IV patients $(n=4)$ as high risk. With Binet stages, only stage A patients $(n=29)$ and stage $\mathrm{B}$ and $\mathrm{C}$ patients $(n=5)$ were distinguished. To compare the mean expression levels of the groups, a Welch two-sample $t$-test was used in the R statistical environment.

\subsection{Primary CLL RNA Samples}

RNA isolated from peripheral blood mononuclear cells of 34 CLL patients were used for gene expression analyses. All patients consented, and the study had ethical approval, research ethics committee: NUI Galway, permission date: 01-06-2015 (duration: 60 months); reference number: CA01355. A summary of the patient clinical data is provided in Table S3.

\subsection{Reverse Transcription-Coupled Quantitative Real-Time Polymerase Chain Reaction}

Reverse transcription of patient RNA samples was performed using $1 \mu \mathrm{g}$ of RNA sample with Superscript II (Invitrogen, Waltham, MA, USA), according to the manufacturer's instructions. The expression of genes was analyzed by using PrimeTime Predesigned qPCR assays (Integrated 
DNA Technologies, IDT, Coralville, IA, USA). Abelson murine leukemia viral oncogene homolog 1 (ABL1) was used as a housekeeping gene for normalization. The TaqMan primer and probe sets for all genes (ABL1, ZAP70, and DNPEP) were predesigned sets purchased from Integrated DNA Technology. For the quantitative polymerase chain reaction (qPCR), Agilent Brilliant III qPCR master mix was used. Each qPCR was performed in a $6-\mu \mathrm{L}$ reaction volume containing $0.3 \mu \mathrm{L}$ of cDNA, $2.1-\mu \mathrm{L} \mathrm{H}_{2} \mathrm{O}, 3.0 \mu \mathrm{L}$ of qPCR master mix, and $0.6 \mu \mathrm{L}$ of the qPCR primer assay. Initial denaturation at $95^{\circ} \mathrm{C}$ for $15 \mathrm{~min}$ was followed by 40 cycles of a denaturation step at $95^{\circ} \mathrm{C}$ for $15 \mathrm{~s}$, an annealing step at $57.5^{\circ} \mathrm{C}$ for $30 \mathrm{~s}$, and an extension step at $72{ }^{\circ} \mathrm{C}$ for $30 \mathrm{~s}$ on a Roche LC480 qPCR light cycler.

The threshold cycle $(\mathrm{Ct})$ value was calculated as the cycle number at which the fluorescence of the reporter reached a fixed threshold. The calculation of the relative expression of the target genes in comparison to the reference gene was performed in an $\mathrm{R}$ statistical environment (Supplementary R-Script).

\subsection{Cell Culture}

The Mec-1 CLL cell line was cultured in RPMI-1640 medium (Sigma, St. Louis, MO, USA) containing 10\% heat-inactivated FBS (Hyclone, Marlborough, MA, USA), penicillin (100 U/mL), streptomycin $(100 \mu \mathrm{g} / \mathrm{mL})$ (Sigma), and L-glutamine $(2 \mathrm{mM})$. The bone marrow mesenchymal stromal cell line, HS-5, was cultured in DMEM (high-glucose, Gibco, Waltham, MA, USA) supplemented with 10\% FBS (Hyclone), GlutaMAX-I (2 mM) (Gibco), penicillin (100 U/mL), streptomycin $(100 \mu \mathrm{g} / \mathrm{mL})$, and 1-mM sodium pyruvate (Sigma). Primary CLL samples were generated from peripheral blood samples by isolating the mononuclear cell (MNC) fraction with Ficoll gradient-centrifugation [32]. MNCs were stored in liquid nitrogen until use. Upon revival of the cells, viability was determined with trypan blue staining. Only samples with viability above $60 \%$ were used. All patients consented according to the local Ethical Committee Regulations. Primary CLL cells were grown on an HS-5 stromal feeder layer in RPMI-1640 medium (Sigma) containing 10\% FBS, penicillin (100 U/mL), streptomycin $(100 \mu \mathrm{g} / \mathrm{mL})$, and L-glutamine $(2 \mathrm{mM})$. The HS-5 cells were stably transfected with GFP (green fluorescent protein) to enable their identification and separation from the CLL cell population in downstream analyses.

\subsection{Measurement of Cell Viability}

Mec-1 cell viability was quantified with annexin $\mathrm{V}$ staining. Cells were collected and stained with annexin V-APC (Immunotools) in annexin V buffer (10-mM HEPES/NaOH, pH 7.5, 140-mM $\mathrm{NaCl}$, and 2.5-mM $\mathrm{CaCl}_{2}$ ) for $15 \mathrm{~min}$ on ice in the dark. Samples were analyzed on a FACS Canto II flow cytometer. The viability of primary CLL cells was determined using the viability dye, To-Pro-3 (Molecular Probes), according to the manufacturer's protocols. The samples were analyzed using the BD FACS Canto II flow cytometer (BD Bioscience, San Diego, CA, USA) by collecting 30,000 events. HS-5 cells were excluded from the analysis by gating out $\mathrm{GFP}^{+} / \mathrm{FSC}^{\text {high }}$ events. Statistical analysis was performed using FCSExpress (DeNovo Software Inc., Pasadena CA, USA) and GraphPad Prism (GraphPad Software Inc., La Jolla, San Diego, CA, USA) software packages.

\subsection{DNPEP Enzyme Activity Assay}

L-aspartic acid 7-amido-4-methylcoumarin (Asp-AMC) was used as the substrate to analyze the DNPEP enzyme activity. Mec-1 cell lysate, as the source of DNPEP enzyme, was obtained by lysing $1 \times 10^{6}$ Mec-1 cells in $100 \mathrm{uL}$ of cell lysis buffer (250- $\mu$ M HEPES, $\mathrm{pH} 7.4,25-\mu \mathrm{M}$ CHAPS, and $2-\mu \mathrm{M}$ DTT). Ten microliters of cell lysate was dispensed into a black, clear-bottom 96-well plate and incubated with $100-\mu \mathrm{L}$ assay buffer (50-mM Tris- $\mathrm{HCl}, \mathrm{pH} 7.5)$ containing $22-\mu \mathrm{M}$ ASP-AMC substrate at $37^{\circ} \mathrm{C}$; As a negative control, DNPEP activity was blocked by adding $0.5-\mathrm{mM} \mathrm{ZnCl}_{2}$ to the reaction. DNPEP activity was monitored kinetically by determining the fluorescence produced in every $60 \mathrm{~s}$ over $30 \mathrm{~min}$. Enzyme activity was calculated from the linear portion of the fluorescent intensity graphs. The effect of the DNPEP inhibitor (DI93293) was calculated according to the following formula: activity score 
$=\left(\left(\mathrm{RFI}_{\mathrm{DI}}-\mathrm{RFI}_{\mathrm{u}}\right) /\left(\mathrm{RFI}_{\mathrm{u}}-\mathrm{RFI}_{Z \mathrm{n}}\right)\right) \times 100 \%$, where $\mathrm{RFI}_{\mathrm{u}}$ : enzyme activity of untreated sample, $\mathrm{RFI}_{\mathrm{DI}}$ : enzyme activity of DI-treated sample, and $\mathrm{RFI}_{\mathrm{Zn}}$ : same of sample containing $\mathrm{ZnCl}_{2}$.

\section{Conclusions}

In conclusion, by using gene co-expression analysis, we identified DNPEP as a biomarker to identify CLL patients with poor prognosis. This biomarker can predict and stratify the risk of when CLL patients develop the progressive disease, requiring therapy. This is comparable to the Rai and Binet clinical CLL staging systems. DNPEP, in combination with IgHV, could be used as a prognostic tool for when CLL patients develop the progressive disease, requiring therapy. Furthermore, our results warrant further study evaluations of DNPEP as a novel target for combination therapy to reverse drug resistance.

Supplementary Materials: The following are available online at http://www.mdpi.com/2072-6694/12/7/1876/s1. Figure S1: Effect of BCR-kinase correlating genes in time to treatment and overall survival probability of CLL patients, Figure S2: DNPEP inhibition by DI93293 kinetic assay, Table S1: Databases and selected samples for co-expression analysis, Table S2: List of genes correlating with ZAP-70 and at least two other BCR-signaling kinase genes, Table S3: Clinical data of patient samples used for qRT-PCRs, Supplementary R-Script: Normalization of Ct values.

Author Contributions: Conceptualization, C.O. and E.S.; Data curation, N.P.; Funding acquisition, E.S.; Investigation, P.K., S.M., K.A., C.O. and E.S.; Methodology, P.K., S.M., K.A., M.A.M., C.O. and E.S.; Project administration, C.O.; Resources, N.P.; Software, M.A.M.; Supervision, C.O. and E.S.; Visualization, P.K. and S.K.; Writing—original draft, P.K., S.M. and E.S.; Writing—review \& editing, S.K. and E.S. All authors have read and agreed to the published version of the manuscript.

Funding: This research was funded by the Irish Cancer Society and Science Foundation Ireland (BCNI, 14/ICS/B3042).

Acknowledgments: The DNPEP inhibitor (DI93293) was a kind gift from Philip Kiser, currently affiliated with the University of California Irvine. The independent dataset used, taken from 107 CLL patients, was collected by Tobias Herold (https://www.ncbi.nlm.nih.gov/geo/query/acc.cgi?acc=GSE22762) et al. [17].

Conflicts of Interest: The authors declare no conflict of interest.

\section{References}

1. Gill, J.; Hughes, M.E. Primer on Chronic Lymphocytic Leukemia: Part I. J. Nurse Pract. 2018, 14, 302-308. [CrossRef]

2. Andreeff, M.; Darzynkiewicz, Z.; Sharpless, T.K.; Clarkson, B.D.; Melamed, M.R. Discrimination of human leukemia subtypes by flow cytometric analysis of cellular DNA and RNA. Blood 1980, 55, 282-293. [CrossRef] [PubMed]

3. Hamblin, T.J.; Davis, Z.; Gardiner, A.; Oscier, D.G.; Stevenson, F.K. Unmutated Ig V(H) genes are associated with a more aggressive form of chronic lymphocytic leukemia. Blood 1999, 94, 1848-1854. [CrossRef] [PubMed]

4. Dal Porto, J.M.; Gauld, S.B.; Merrell, K.T.; Mills, D.; Pugh-Bernard, A.E.; Cambier, J. B cell antigen receptor signaling 101. Mol. Immunol. 2004, 41, 599-613. [CrossRef]

5. Chen, L.; Widhopf, G.; Huynh, L.; Rassenti, L.; Rai, K.R.; Weiss, A.; Kipps, T.J. Expression of ZAP-70 is associated with increased B-cell receptor signaling in chronic lymphocytic leukemia. Blood 2002, 100, 4609-4614. [CrossRef]

6. Kim, S.Z.; Chow, K.U.; Kukoc-Zivojnov, N.; Boehrer, S.; Brieger, A.; Steimle-Grauer, S.A.; Harder, L.; Hoelzer, D.; Mitrou, P.S.; Weidmann, E. Expression of ZAP-70 Protein Correlates with Disease Stage in Chronic Lymphocytic Leukemia and is Associated with, but not Generally Restricted to, Non-mutated Ig V H Status. Leuk. Lymphoma 2004, 45, 2037-2045. [CrossRef]

7. Wiestner, A.; Rosenwald, A.; Barry, T.S.; Wright, G.; Davis, R.E.; Henrickson, S.E.; Zhao, H.; Ibbotson, R.E.; Orchard, J.A.; Davis, Z.; et al. ZAP-70 expression identifies a chronic lymphocytic leukemia subtype with unmutated immunoglobulin genes, inferior clinical outcome, and distinct gene expression profile. Blood 2003, 101, 4944-4951. [CrossRef] 
8. Chen, C.C.; Manning, A.M. Transcriptional regulation of endothelial cell adhesion molecules: A dominant role for NF-kappa B. Agents Actions Suppl. 1995, 47, 135-141.

9. Kopp, E.B.; Ghosh, S. NF-kappa B and rel proteins in innate immunity. Adv. Immunol. 1995, 58, 1-27.

10. Kröber, A.; Bloehdorn, J.; Hafner, S.; Bühler, A.; Seiler, T.; Kienle, D.; Winkler, D.; Bangerter, M.; Schlenk, R.F.; Benner, A.; et al. Additional genetic high-risk features such as 11q deletion, 17p deletion, and V3-21 usage characterize discordance of ZAP-70 and VH mutation status in chronic lymphocytic leukemia. J. Clin. Oncol. 2006, 24, 969-975. [CrossRef]

11. Chaar, B.T.; Schergen, A.K.; Grosso, L.E. Discordance of ZAP-70 in patients with chronic lymphocytic leukemia. Int. J. Lab. Hematol. 2008, 30, 36-40. [CrossRef] [PubMed]

12. Gobessi, S.; Laurenti, L.; Longo, P.G.; Sica, S.; Leone, G.; Efremov, D.G. ZAP-70 enhances B-cell-receptor signaling despite absent or inefficient tyrosine kinase activation in chronic lymphocytic leukemia and lymphoma B cells. Blood 2007, 109, 2032-2039. [CrossRef]

13. Brown, J.R.; Byrd, J.C.; Coutre, S.E.; Benson, D.M.; Flinn, I.W.; Wagner-Johnston, N.D.; Spurgeon, S.E.; Kahl, B.S.; Bello, C.; Webb, H.K.; et al. Idelalisib, an inhibitor of phosphatidylinositol 3-kinase p1108, for relapsed/refractory chronic lymphocytic leukemia. Blood 2014, 123, 3390-3397. [CrossRef] [PubMed]

14. Pekarsky, Y.; Zanesi, N.; Croce, C.M. Molecular basis of CLL. Semin. Cancer Biol. 2010, 20, 370-376. [CrossRef]

15. Furman, R.; Asgary, Z.; Mascarenhas, J.; Liou, H.; Schattner, E. Modulation of NF-kappa B activity and apoptosis in chronic lymphocytic leukemia B cells. J. Immunol. 2000, 164, 2200-2206. [CrossRef]

16. Papakonstantinou, N.; Ntoufa, S.; Chartomatsidou, E.; Kotta, K.; Agathangelidis, A.; Giassafaki, L.; Karamanli, T.; Bele, P.; Moysiadis, T.; Baliakas, P.; et al. The histone methyltransferase EZH2 as novel prosurvival factor in clinically aggressive chronic lymphocytic leukemia. Oncotarget 2016, 14, 35946-35959. [CrossRef]

17. Herold, T.; Jurinovic, V.; Metzeler, K.H.; Boulesteix, A.L.; Bergmann, M.; Seiler, T.; Mulaw, M.; Thoene, S.; Dufour, A.; Pasalic, Z.; et al. An eight-gene expression signature for the prediction of survival and time to treatment in chronic lymphocytic leukemia. Leukemia 2011, 25, 1639. [CrossRef]

18. Chen, Y.; Farquhar, E.; Chance, M.; Palczewski, K.; Kiser, P. Insights into Substrate Specificity and Metal Activation of Mammalian Tetrahedral Aspartyl Aminopeptidase. J. Biol. Chem. 2012, 287, 13356-13370. [CrossRef]

19. Chen, Y.; Tang, H.; Seibel, W.; Papoian, R.; Oh, K.; Li, X.; Zhang, J.; Golczak, M.; Palczewski, K.; Kiser, P.D. Identification and Characterization of Novel Inhibitors of Mammalian Aspartyl Aminopeptidase. Mol. Pharmacol. 2014, 86, 231-242. [CrossRef]

20. Kehlen, A.; Lendeckel, U.; Dralle, H.; Langner, J.; Hoang-Vu, C. Biological significance of aminopeptidase N/CD13 in thyroid carcinomas. Cancer Res. 2003, 63, 8500-8506.

21. Surowiak, P.; Drag, M.; Materna, V.; Suchocki, S.; Grzywa, R.; Spaczyński, M.; Dietel, M.; Oleksyszyn, J.; Zabel, M.; Lage, H. Expression of aminopeptidase N/CD13 in human ovarian cancers. Int. J. Gynecol. Cancer 2006, 16, 1783-1788. [CrossRef] [PubMed]

22. Severini, G.; Gentilini, L.; Tirelli, C. Diagnostic evaluation of alanine aminopeptidase as serum marker for detecting cancer. Cancer Biochem. Biophys. 1991, 12, 199-204.

23. Martínez, J.M.; Prieto, I.; Ramírez, M.J.; Cueva, C.; Alba, F.; Ramírez, M. Aminopeptidase activities in breast cancer tissue. Clin. Chem. 1999, 45, 1797-1802. [CrossRef]

24. Hashida, H.; Takabayashi, A.; Kanai, M.; Adachi, M.; Kondo, K.; Kohno, N.; Yamaoka, Y.; Miyake, M. Aminopeptidase $\mathrm{N}$ is involved in cell motility and angiogenesis: Its clinical significance in human colon cancer. Gastroenterology 2002, 122, 376-386. [CrossRef] [PubMed]

25. Hitzerd, S.M.; Verbrugge, C.S.E.; Ossenkoppele, G.J.; Jansen, G.; Peters, G.J. Positioning of aminopeptidase inhibitors in next generation cancer therapy. Amino Acids 2014, 46, 793-808. [CrossRef] [PubMed]

26. Scornik, O.A.; Botbol, V. Bestatin as an experimental tool in mammals. Curr. Drug Metab. 2001, 2, 67-85. [CrossRef] [PubMed]

27. Krige, D.; Needham, L.A.; Bawden, L.J.; Flores, N.; Farmer, H.; Miles, L.E.; Stone, E.; Callaghan, J.; Chandler, S.; Clark, V.L.; et al. CHR-2797: An antiproliferative aminopeptidase inhibitor that leads to amino acid deprivation in human leukemic cells. Cancer Res. 2008, 68, 6669-6679. [CrossRef]

28. Matre, P.; Velez, J.; Jacamo, R.; Qi, Y.; Su, X.; Cai, T.; Chan, S.M.; Lodi, A.; Sweeney, S.R.; Ma, H.; et al. Inhibiting glutaminase in acute myeloid leukemia: Metabolic dependency of selected AML subtypes. Oncotarget 2016, 7, 79722-79735. [CrossRef] 
29. Langfelder, P.; Horvath, S. WGCNA: An R package for weighted correlation network analysis. BMC Bioinform. 2008, 9, 559. [CrossRef]

30. Aguirre-Gamboa, R.; Gomez-Rueda, H.; Martínez-Ledesma, E.; Martínez-Torteya, A.; Chacolla-Huaringa, R.; Rodriguez-Barrientos, A.; Tamez-Peña, J.G.; Treviño, V. SurvExpress: An online biomarker validation tool and database for cancer gene expression data using survival analysis. PLoS ONE 2013, 8, e74250. [CrossRef]

31. Jalali, A.; Alvarez-Iglesias, A.; Roshan, D.; Newell, J. Visualising statistical models using dynamic nomograms. PLoS ONE 2019, 14, e0225253. [CrossRef]

32. Baev, D.V.; Krawczyk, J.; O’Dwyer, M.; Szegezdi, E. The BH3-mimetic ABT-737 effectively kills acute myeloid leukemia initiating cells. Leuk. Res. Rep. 2014, 3, 79-82. [CrossRef]

(C) 2020 by the authors. Licensee MDPI, Basel, Switzerland. This article is an open access article distributed under the terms and conditions of the Creative Commons Attribution (CC BY) license (http://creativecommons.org/licenses/by/4.0/). 